\title{
Benefícios dos grupos no manejo da hipertensão arterial sistêmica: percepções de pacientes e médicos
}

\author{
Benefits of groups in managing systemic arterial hypertension: perceptions of patients and physicians
}

\author{
Beneficios de los grupos en el manejo de la hipertensión arterial sistémica: \\ percepciones de los pacientes y de los médicos
}

Rodrigo Pereira do Amaral. Universidade Federal de Santa Catarina (UFSC). Florianópolis, SC, Brasil. rp_amaral@hotmail.com Charles Dalcanale Tesser. Universidade Federal de Santa Catarina (UFSC). Florianópolis, SC, Brasil. charles.tesser@ufsc.br (Autor correspondente) Pedro Müller. Universidade Federal de Santa Catarina (UFSC). Florianópolis, SC, Brasil. pedro.muller.18@gmail.com

\section{Resumo}

Objetivo: investigar a natureza dos benefícios da utilização de grupos nos serviços de atenção primária à saúde no manejo da hipertensão arterial, sob a ótica de pacientes e médicos participantes. Métodos: estudo qualitativo descritivo por meio de entrevistas semiestruturadas com pacientes e médicos participantes de grupos distintos consolidados, selecionados intencionalmente, incluídos em pares médico-paciente, até ser percebida saturação nos dados. As entrevistas foram submetidas à análise temática. Resultados e discussão: a análise das entrevistas indicou benefícios em quatro campos: educação em saúde, adesão, apoio psicossocial e melhora na qualidade de vida. Efeitos promotores de saúde foram percebidos pelos participantes, embora restritos ao nível individual e comunitário. Os grupos atenuam a "inadequação comportamental" da estratégia preventiva de alto risco, segundo Geoffrey Rose, base do manejo atual da hipertensão. Também melhoram e facilitam a função educativa dos profissionais de saúde, auxiliando na adesão e incrementando significativamente 0 apoio social dos pacientes. Conclusões: a utilização dos grupos de hipertensos pode melhorar o manejo da hipertensão e promover a saúde dos envolvidos e, se conduzidos de forma dialogal e participativa, seus benefícios podem ser amplificados.

\section{Abstract}

Objective: To investigate the nature of the benefits of using groups within primary care services to manage hypertension, from the point of view of both patients and physicians. Methods: A qualitative descriptive study using semi-structured interviews with patients and doctors attending distinct consolidated groups, which have been purposely selected and carried out in physician-patient pairs until reaching data saturation. The interviews were subjected to thematic analysis. Results and discussion: The analysis of the interviews showed benefits in four fields: health education, compliance, psychosocial support, and quality of life improvement. Health promoting effects were perceived by participants, although restricted to individual and community levels. Participation in groups attenuates the "behavioral inadequacy" of high-risk preventive strategy, according to Geoffrey Rose, based on current management of hypertension. It also improves and facilitates health professionals' educational role, improving compliance and significantly increasing social support for patients. Conclusions: The use of groups for hypertensive patients can improve hypertension management and promote the health of those involved. These benefits can be amplified if management is conducted in a dialogical and participatory way.

\section{Resumen}

Objetivo: investigar la naturaleza de los beneficios de la utilización de grupos en los servicios de atención primaria a la salud en el manejo de la hipertensión arterial sistémica, desde la óptica de los pacientes y de los médicos participantes. Métodos: se realizó un estudio descriptivo a través de entrevistas semiestructuradas con pacientes y médicos de diferentes grupos consolidados, seleccionados intencionalmente, incluidos en pares médico-paciente hasta que se percibe una saturación en los datos. Las entrevistas fueron sometidas a análisis temático. Resultados y discusión: el análisis indicó beneficios en cuatro áreas: educación para la salud, cooperación del paciente, apoyo psicosocial y mejora en la calidad de vida. Se observaron efectos promovedores de salud, aunque limitados a nivel individual y comunitario. Los grupos atenúan la "inadecuación comportamental" de la estrategia preventiva de alto riesgo, según Geoffrey Rose, base del manejo actual de la hipertensión. Además, mejoran y facilitan la función educativa de los profesionales de la salud, auxiliando en la adhesión e incrementando significativamente el apoyo social de los pacientes. Conclusiones: el uso de grupos puede mejorar el manejo de la hipertensión y promover la salud de las personas involucradas, si se realiza de manera dialógica y participativa.

Como citar: Amaral RP, Tesser CD, Müller P. Benefícios dos grupos no manejo da hipertensão arterial sistêmica: percepções de pacientes e médicos. Rev Bras Med Fam Comunidade. 2013;8(28:196-202. Disponível em: http://dx.doi.org/10.5712/rbmfc8(28)762
Palavras-chave: Atenção Primária à Saúde Educação em Saúde Hipertensão Grupos de Autoajuda

\section{Keywords:} Primary Health Care Health Education Hypertension Self-Help Groups

Palabras clave: Atención Primaria de Salud Educación en Salud Hipertensión Grupos de Autoayuda 


\section{Introdução}

O manejo da hipertensão arterial sistêmica nos indivíduos é complexo e problemático no mundo todo, pois compreende a farmacoterapia, o controle de peso, a adoção de hábitos alimentares saudáveis - redução do sódio na dieta e menor consumo de bebidas alcoólicas - bem como, o abandono do tabagismo e a prática de atividade física regular ${ }^{1}$. Estima-se que metade dos hipertensos sabe de sua condição (dos quais, metade faz tratamento) e dois terços dos hipertensos em seguimento náo têm valores pressóricos adequados. As dificuldades no manejo envolvem a pouca adesão, aspectos psicológicos e sociais relacionados aos serviços (organização, estrutura, acesso e características da relação médico-paciente) e outras razôes (ausência de sintomas, normalização da pressão, consumo de álcool, etc. $)^{2-5}$.

Uma tentativa de melhorar o manejo desses pacientes foi a utilização de grupos, complementando atendimentos individuais. O "North Karelia Project" ajudou a reduzir os fatores de risco de uma populaçáo finlandesa, diminuindo significativamente a mortalidade por doenças cardiovasculares em 22 anos de programa. Kulčar ${ }^{7}$, acompanhando por 10 anos hipertensos membros permanentes de grupos, constatou redução na mortalidade e aumento da expectativa de vida, quando comparados aos membros temporários e controles.

Atualmente, a expressiva prevalência e o escasso ou nulo benefício do uso de drogas nos hipertensos leves ${ }^{8}$ (a maioria dos hipertensos), ressalta a relevância do estudo de recursos de cuidado e orientação para esse problema. Por outro lado, grupos voltados a hipertensos na atençáo primária à saúde (APS) sáo muito comuns no Brasil, podendo obter resultados relevantes, porém são pouco pesquisados, e estudos avaliativos como os anteriormente mencionados mostram resultados significativos ${ }^{9}$. Todavia, mais raros ainda são estudos investigando potenciais benefícios ocorridos durante e com a participação dos hipertensos nos grupos, não quanto a desfechos clínicos finais, mas em termos de processo de cuidado e autocuidado. Existindo, tais benefícios são observados pelos próprios profissionais e usuários, considerados, nesse contexto, informantes privilegiados.

O objetivo desta pesquisa foi investigar a natureza dos benefícios da utilização de grupos no manejo de pacientes hipertensos na APS, percebidos por médicos e pacientes participantes destes grupos, não quanto a desfechos clínicos, mas em termos processuais no cuidado e autocuidado.

\section{Métodos}

Foram investigados pacientes hipertensos e profissionais participantes de grupos de hipertensão selecionados intencionalmente. Os critérios de seleção incluíram participantes de grupos consolidados e estáveis, considerando tais sujeitos informantes privilegiados. Fez-se um levantamento nos Centros de Saúde (CS) de Florianópolis-SC em 2007, que mostrou haver grupos em 21 CS (45\% do total). Médicos coordenavam ou participavam de 20 (95\%) deles. Foram incluídos grupos com reuniôes regulares há pelo menos um ano, com um médico atuante por pelo menos seis meses, que já tivesse trabalhado com grupos por mais de dois anos. Apenas um médico (com maior experiência) foi selecionado por grupo. Foram selecionados participantes dos 16 grupos incluídos, requisitando-se, para cada médico entrevistado, a indicação de três pacientes hipertensos que, segundo sua avaliação, houvessem obtido bons resultados com a participação e frequentassem o grupo regularmente há pelo menos um ano. Um desses três pacientes foi convidado aleatoriamente; em caso de recusa ou ausência, convidava-se outro. Considerou-se inicialmente que um médico e um paciente de cada grupo contemplariam perspectivas distintas (de profissionais e usuários) sobre as experiências e seguiu-se essa ordem na coleta de dados, entrevistando-se sequencialmente e separadamente um par médico-paciente de cada grupo, nos serviços ou nas residências dos pacientes.

As entrevistas foram transcritas imediatamente depois de realizadas e pré-analisadas. Observou-se frequente repetição de conteúdos, atingindo-se saturação nos dados ${ }^{10} \mathrm{com}$ as vinte primeiras entrevistas (um médico e um paciente dos dez primeiros grupos investigados).

As entrevistas seguiram um roteiro semiestruturado, com perguntas abertas, e constituíram o corpus da pesquisa, submetido à análise de conteúdo, técnica de análise temática ${ }^{11}$. A pesquisa foi aprovada pelo Comitê de Ética em Pesquisa da Universidade Federal de Santa Catarina (UFSC), protocolo processo 325 FR 113549. 


\section{Resultados e discussão}

Dos médicos entrevistados, 6 eram mulheres e 4 eram homens. Cinco possuíam entre 3 e 5 anos de experiência com grupos; 2, entre 6 e 10 anos; 2, entre 11 e 20 anos; e 1, mais de 20 anos. Dos 10 usuários entrevistados, 9 eram mulheres. Três possuíam renda mensal familiar entre 0 e 2 salários mínimos; 5, entre 3 e 4 salários mínimos; e 2, mais de 4 salários mínimos. Um era analfabeto; 7 tinham ensino fundamental incompleto; e 2, ensino fundamental completo. A idade média dos usuários foi 67,2 anos, com tempo médio de diagnóstico de hipertensão de 13,7 anos e média de participação em grupos de 5,3 anos. Percebeu-se significativa experiência com os grupos nos profissionais, indicando coerência nos critérios de seleção, assim como expressiva dominância feminina nos grupos, como é comum. A análise das entrevistas permitiu reconhecer benefícios percebidos pelos entrevistados nos seguintes campos temáticos: educaçáo em saúde, adesão, apoio psicossocial e qualidade de vida.

\section{Educação em saúde}

O curso comumente assintomático da hipertensão, aliado à falta de conhecimento, está relacionado ao fato de muitos pacientes não compreenderem bem um tratamento para tal condição: "Eu achava, assim, que era uma coisa normal [...] $o$ negócio da hipertensão" (paciente 1=P1). "Eu não sinto essa pressão [...], se a gente sentisse o que é a diferença, a gente já sabia, [...], mas a gente não sente" (P6). Cotta et al. ${ }^{12}$ mostraram baixos padrôes de conhecimento dos hipertensos sobre sua condição e também sugeriram açóes grupais para enfrentamento do problema.

Os grupos podem ser utilizados como ferramenta para melhorar a educação em saúde, uma vez que a forma como a informação é ou pode ser trabalhada neles apresenta vantagens em relação à consulta individual em diversos aspectos, segundo profissionais entrevistados. Dispóem de mais tempo para orientaçôes e, simultaneamente, por falarem para um número maior de pessoas, gastam menos tempo: A grande vantagem [...] é que tu podes atuar de uma forma muito mais ampla e num curto periodo de tempo, de uma hora, duas... atingindo trinta a quarenta pessoas, e isso com certeza vale mais que uma consulta no consultório de dez a quinze minutos (Médico 8 = M8).

A educação em saúde tem sido hegemonicamente entendida como transmissão de informações e prescrições, visando ao controle pressórico e das condutas consideradas fatores de risco, tanto no cuidado clínico individual como nos encontros grupais ${ }^{13}$. Nessa lógica, a informaçáo correta somada ao empenho do paciente (sua disciplina, vontade e adesão) resultaria em comportamento adequado. Esta visão se mostrou equivocada nas últimas décadas, notadamente a partir das tentativas de prevenção da AIDS ${ }^{14}$. Apesar de haver movimentos buscando superar esse pressuposto e as práticas educativas unidirecionais, não se nega que a informação é necessária. No caso da hipertensão, os grupos foram identificados como valiosos, nesse aspecto, pelos entrevistados. Os entrevistados relataram aumento do nível de conhecimento dos hipertensos com a participação nos grupos: "Eles ficam mais conscientes depois que eles entendem mais como é que funciona o corpo" (M10); "Näo aprende quem não quer. Isso é bem explicadinho" (P8).

Vantagem adicional é o caráter descontraído da forma como o conhecimento pode ser trabalhado, sendo que a presença de outros pacientes estimula o debate e faz surgir dúvidas, desde que a dinâmica do grupo permita e estimule: Às vezes uma coisa que a pessoa näo pergunta na consulta vai aparecer a dúvida em casa [...]. E no grupo há essa oportunidade, é uma coisa um pouco mais solta, esses questionamentos aparecem [...] em forma de curiosidade, às vezes na forma de uma brincadeira. E então as pessoas vêm com um outra ideia [...], não vêm com aquela tensão da consulta, e não tem aquele compromisso como se fosse uma receita (M3).

Esse é um aspecto importante do ponto de vista educacional: a troca de experiências nos grupos entre os participantes: "[...] às vezes tem alguém que já tem alguma complicação da hipertensão e ai tem tudo assim, como é que sentem, tinha dor assim, uma dor tal, como é que fez pra melhorar, como é que passou aquela situação..., sempre vem à tona" (M7). Geoffrey Rose $\mathrm{e}^{15}$ já enfatizava que a simples menção da prevenção de problemas futuros é pouco mobilizadora de mudanças de hábitos presentes e da adesão a um tratamento crônico. Os grupos permitem aos pacientes terem contato e dividirem saberes e experiências com outros que vivem limitaçóes impostas pelas complicaçóes relacionadas à hipertensão, o que dá aos primeiros a oportunidade de constatar, de forma indireta, esses problemas, facilitando a percepção e o envolvimento emocional e psicológico com o tema de modo a torná-lo algo mais concreto, além de trabalhar a informação; achado também relatado por Ferreira Neto e Kind $^{16}$. A exploração e debate de exemplos, problemas e soluçôes pertencentes à realidade dos indivíduos, instigando-os a participar do processo de forma ativa, aproxima-se do proposto pela "educação problematizadora", defendida por Freire ${ }^{17}$. 
Uma educação emancipadora, tem sido discutida na APS e pelo movimento brasileiro da educação popular em saúde ${ }^{18,19}$, convergindo com recentes estudos sobre cuidado e autocuidado em situaçóes crônicas na ênfase que dão ao aspecto dialogal, participativo e reflexivo da troca de experiências e perspectivas dos educandos ${ }^{20}$. Também convergem na constatação de que a transmissão de conhecimentos é insuficiente para lograr êxito educativo e substancial contribuição para o aumento da autonomia relativa e autocuidado, bem como na necessidade de discussão a partir das realidades e saberes da vida e do cuidado dos usuários, contrastando com a tendência monologal e de transmissão unidirecional de conhecimento comum nos profissionais, aproximada ao que Freire ${ }^{17}$ chamou de educação bancária.

Por outro lado, os achados do presente estudo sugerem que os grupos podem amenizar um dos graves problemas inerentes ao que Rose ${ }^{15}$ chamou de estratégia preventiva de alto risco, cuja característica é o foco no cuidado individual da fração populacional de maior risco (hipertensos). Para Rose ${ }^{15}$, essa estratégia é comportamentalmente inadequada, pois demanda mudança de hábitos em indivíduos inseridos em um meio social que desestimula tais mudanças. O incentivo ao desenvolvimento de uma rede de relacionamentos entre pessoas com riscos, problemas e dificuldades semelhantes, facilitado e induzido pelos grupos, representa uma alternativa que atenua o problema da inadequaçáo comportamental citada, aumentando o apoio social aos cuidados e mudanças. Nos grupos e nas redes de relaçóes por eles fomentadas, os pacientes apreendem, reconstroem e manejam informaçóes e situações de forma melhor quando colocadas por alguém que vive a mesma realidade. Isso aparece indiretamente no relato de $\mathrm{P} 5$, falando de pessoa que não frequentava o grupo: "Ela é descompensada. Se ela fosse [no grupo], tinha mais ânimo. Ela via aquelas pessoas falarem o que fazem, o que comem, ai é fácil, porque de repente você descobre alguma coisa que pode aproveitar" (P5).

Como a estratégia de alto risco, apesar de suas limitaçóes e problemas, é hoje o centro da atuação nas desordens cardiovasculares, seu aperfeiçoamento deve ser buscado e os grupos podem ser uma via nesse sentido. Isso reforça a necessidade de uma abordagem dialogal e participativa nos grupos, chamada por Alves ${ }^{13}$ de modelo dialógico, que procura trazer à tona, compartilhar e refletir sobre as concepçóes, dificuldades, vivências, práticas e realidades dos usuários, avançando na superaçáo da herança autoritária e controladora nas relaçóes dos profissionais com os pacientes ${ }^{21}$.

Outras vantagens da abordagem grupal, mencionadas nas entrevistas, sinérgicas com as anteriores, são a possibilidade de utilizar dinâmicas e brincadeiras que cumprem múltiplos propósitos como educação, socialização e lazer, aumentando a adesão ao grupo e a apreensão de conteúdos. Os grupos também estimulam o autocuidado, uma vez que em muitos encontros são realizados exercícios físicos, aferição da pressão arterial e estímulo a uma alimentação adequada: " $A$ gente fez apresentação de casos, bem comuns do dia a dia deles, em forma de teatro. Todo mundo participou, se vestiram a caráter, com fantasia e tal. Eles adoraram" (M10). "A gente estimula o pessoal a trazer chá, trazer algum lanchinho, não trazer muita coisa doce ou trazer dietético, porque tem os diabéticos, [...] utiliza a hora do lanche pra fazer uma orientação de alimentação" (M7). Além disso, houve menção à difusão do conhecimento para fora dos grupos: "As pessoas que frequentam aqui são de mais idade [...] responsáveis por geraçôes, filhos e netos, [...] estão espalhando isso pela comunidade" (M8).

Em síntese, houve unanimidade entre os entrevistados sobre haver aumento do conhecimento e do autocuidado dos usuários frequentadores, responsabilizando-se mais por sua própria saúde.

\section{Adesão}

Para os entrevistados, a participaçáo nos grupos contribui para o aumento da adesão ao tratamento, tanto pelo conhecimento como pelo apoio psicossocial dado pelo grupo: "eles passam a se cuidar mais, com certeza" (M5); "porque às vezes a pessoa sozinha, ela pode ficar desanimada. E ela estando no grupo, ela sempre tá com mais afinco” (P5). Relatam que o grupo também aproxima os pacientes da equipe profissional, aumentando a confiança e o respeito mútuo e, consequentemente, a segurança do paciente em relação às orientaçôes: "Além do relacionamento com a equipe, com profissionais envolvidos, se cria uma amizade que é muito boa" (M2). "Porque tem gente que acha que o médico tá ali em cima de um pedestal, não pode nem falar contigo, $e$ em um grupo, você vê que não é assim" (P5).

A adesão à conduta, medicamentosa ou não, é algo esperado pelo médico. Entretanto, o que se observa muitas vezes é que a relação entre paciente e médico (comumente supondo o primeiro submisso ao segundo) não garante a adesão, especialmente no manejo de condiçôes crônicas assintomáticas como hipertensão e diabetes. A falta de adesão é considerada uma das causas do difícil controle e, muitas vezes, os pacientes são considerados culpados pelo fracasso terapêutico ${ }^{2,3}$. 
Esforços e movimentos têm tentado introduzir novas abordagens na educação e comunicação em saúde, buscando melhor compreensão do "outro" e do que o orienta. Para quem vivencia a situação, será pelas suas representaçôes sociais que se estabelece o sentido de sua experiência e seu comportamento ${ }^{20}$. Porém, não somente as condiçóes sociais influenciam as representaçóes sociais e as práticas, mas estas representaçóes também são refeitas com a experiência vivida ${ }^{22}$ e tomam importante dimensão na hipertensão, similarmente ao diabetes, por se tratar de condiçáo crônica silenciosa que demanda significativo esforço de adaptação às atividades e restriçôes previstas pelo saber médico.

Há consenso sobre a necessidade de envolver o paciente mais ativamente no cuidado, levando em conta sua visão e respeitando-o como sujeito do processo. Em artigo de revisão, Haynes et al. ${ }^{23}$ concluem que várias intervençóes para melhorar a adesão têm apresentado resultados limitados e precisam ser desenvolvidas e adaptadas às características das pessoas e contextos dos serviços, para que sejam efetivas. Cyrino ${ }^{22}$, investigando o autocuidado de adultos e idosos diabéticos - que têm muitos paralelos com o de hipertensos - encontrou que aqueles indivíduos que não tomam as recomendaçóes médicas de modo rígido e "cego" em seu cotidiano (mas o fazem de forma consciente e crítica, buscando um equilíbrio entre as demandas de controle e o modo como querem levar sua vida) referem uma sensação de confiança, com menor culpa, maior aceitação de sua condição e melhor controle glicêmico.

É cada vez mais evidente a noção de que o paciente não é um passivo recebedor de atenção, mas que ele, seus amigos e seus familiares são também provedores de cuidado. Nesse sentido, os grupos investigados foram percebidos pelos entrevistados como fomentadores de uma adesão mais ativa, ao fornecerem amparo emocional, tecnológico e de aconselhamento aos indivíduos que deles fazem parte, além de estimular o apoio social.

\section{Apoio psicossocial}

As entrevistas indicam que a abordagem grupal pode proporcionar apoio psicossocial aos seus integrantes, diminuindo níveis de ansiedade, melhorando a aceitação da condição de hipertenso, promovendo também melhoria da qualidade de vida. A possibilidade de conviver com pessoas com a mesma situação reduz medos e ansiedades diante da incerteza: " $O$ medo de se sentir, talvez, rejeitado pelo meio social, por ter alguma limitaçáo. E o grupo faz com que caia por terra, porque ali a pessoa percebe que não é ele, têm muitas pessoas assim" (M4); "O que mais me motivou é porque o grupo, ali você encontra pessoas com o mesmo problema" (P5). Isso sugere que o grupo cria uma rede de apoio mútuo, que faz seus membros se sentirem amparados, por terem à disposição pessoas que entenderão suas necessidades, além de trazer mais conhecimento. Proporciona maior estabilidade emocional para enfrentar situaçôes com as quais os hipertensos se deparam: "Às vezes a gente tá tão solitária dentro de casa, triste, chega lá, conversa, e a gente escuta, eles também escutam a gente. É muito bom" (P3). "A gente aprende como lidar, porque de primeiro, quando me sentia mal, eu ficava muito nervosa, e agora eu já sei como me controlar, já tenho ideia." (P2).

Ferreira Neto e Kind ${ }^{16}$ encontraram resultados similares quanto à importância das redes de apoio social para a saúde e, nesse sentido, os grupos não devem ser subvalorizados ${ }^{24}$. Propiciar oportunidades de trocas sociais, notadamente entre idosos - em contextos sociais de famílias pequenas e núcleos familiares instáveis, com proeminência de condiçôes crônicas e maior longevidade, além de maior individualismo nas precárias condiçóes sociais e econômicas da maioria da população - é uma importante estratégia de manutençáo da saúde, aumento da resiliência e empoderamento.

\section{Qualidade de vida}

$\mathrm{Na}$ percepção dos entrevistados, os grupos estimulam a socialização de seus integrantes, resultando em ganhos emocionais, melhora do humor e aumento das atividades de lazer. Vários pacientes fazem novas amizades e tendem a se encontrar com maior frequência fora do grupo: "Eu quase não saio de casa; vou lá; é benefício para minha saúde e pra mim. Eu conheço as minhas amigas, elas convidam pra fazer as coisas e eu vou" (P7). "Eu tenho grandes amigos que eu não conhecia. [...] A gente se conhece, se vê na rua, e isso faz bem. A gente conversa, a gente brinca; tudo ali sai. É muito gostoso" (P4). "Tem vez que eles só querem fazer uma reuniāo pra dançar e se divertir. Então teve uma que foi só isso, [...] uma confraternização, com música. Eles adoram e [...] isso também faz parte da promoção da saúde" (M10). Emerge aqui o tema da promoção da saúde, o qual vem sendo ressignificado como um processo de capacitação da comunidade para atuar na melhoria da sua qualidade de vida e saúde, incluindo maior participação no controle deste processo ${ }^{25}$. 
Um dos núcleos da promoção da saúde é o empowerment, traduzido por empoderamento, que abarca pelo menos dois significados: a) Empoderamento Psicológico, relacionado a um sentimento de maior controle sobre a vida, experimentado pelos indivíduos por meio do pertencimento a distintos grupos, melhor autoestima e motivaçáo; e b) Empoderamento Comunitário ou Social, relativo a processos e açóes que afetam a distribuiçáo do poder no âmbito das esferas pessoais, intersubjetivas e políticas, que inclui e transcende o empoderamento psicológico ${ }^{26}$. Os depoimentos sugerem que o empoderamento social foi pouco explorado nos grupos investigados, sendo mais comum o empoderamento psicológico. Esses grupos atuaram com algum êxito em modificar conhecimentos e hábitos de vida e incrementar o apoio social, agindo em indivíduos e suas redes comunitárias, o que é significativo, porém restrito em termos de determinantes sociais ${ }^{25}$. Pode-se considerar que talvez um enfoque promocionista nos grupos (voltados para hipertensos ou náo), cujas características foram sintetizadas por Santos et al. ${ }^{27}$ em suas diferenças do enfoque preventivista, seja superior em efetividade a este último. Esses resultados mostram convergência com os de Ferreira Neto e Kind ${ }^{16}$ quanto aos efeitos encontrados por estes pesquisadores nas práticas grupais (não especificamente relacionadas à hipertensão) mais consolidadas e "avançadas" nos serviços de APS por eles investigados: 1) facilitaçáo do descentramento da patologia; 2) suporte e apoio social; 3) criação de espaços de sociabilidade; 4) troca de conhecimentos entre pacientes e destes com profissionais; 5) fortalecimento e qualificação dos vínculos entre usuários e equipes. A opção por uma via de promoção da saúde pode amplificar os benefícios dos grupos quanto aos ganhos de qualidade de vida, apoio social, participação, autonomia e empoderamento.

Os métodos desta pesquisa não almejaram e não permitiram uma avaliação da qualidade (educativa, empoderadora ou terapêutica) das relações ou das práticas nos grupos investigados, tampouco uma caracterização ou análise de sua condução. Outra limitação é que seu desenho não previu e não permitiu uma abordagem de gênero.

\section{Considerações finais}

Os resultados sugerem que a abordagem grupal pode ser ferramenta importante no manejo da hipertensáo por meio de diversos e complexos processos. Os grupos parecem constituir uma fonte potencial e relevante de atenuação da inadequação comportamental, segundo Rose ${ }^{15}$, envolvida no manejo da hipertensão. Os benefícios relatados indicam que os grupos podem melhorar a função educativa dos serviços de APS, o apoio social e a qualidade de vida desses pacientes. Além disso, a pouca utilização explícita de um enfoque promocional e resultados promissores nessa área sugerem que os grupos teriam tanto ou mais potência se melhor orientados para a promoçáo da saúde.

Se os encontros grupais construírem práticas dialógicas e redes de apoio, valorização e compreensão mútua de saberes e realidades existenciais com relaçóes solidárias e horizontalizadas ${ }^{28}$, os grupos envolvendo hipertensos e usuários com outros problemas de saúde poderiam se tornar úteis dispositivos na APS e em outros contextos assistenciais.

\section{Referências}

1. Chobanian AV, Bakris GL, Black HR, Cushman WC, Green LA, Izzo JL Jr, et al. The Seventh Report of the Joint National Comittee on Prevention, Detection, Evaluation and Treatment of High Blood Pressure: the JNC 7 report. JAMA. 2003; 289(19): 2560-72. PMid:12748199. http://dx.doi. org/10.1001/jama.289.19.2560

2. European Society of Hypertension, European Society of Cardiology Guidelines Committee. 2003 European Society of Hypertension-European Society of Cardiology guidelines for the management of arterial hypertension. J Hypertens. 2003; 21(6): 1011-1053. PMid:12777938. http://dx.doi. org/10.1097/00004872-200306000-00001

3. Duarte MTC, Cyrino AP, Cerqueira ATAR, Nemes MIB, lyda, M. Motivos do abandono do seguimento médico no cuidado a portadores de hipertensão arterial: a perspectiva do sujeito. Ciênc Saúde Coletiva. 2010;15(5):2603-2610. http://dx.doi.org/10.1590/S1413-81232010000500034

4. Souza MLP, Garnello L. "É muito dificultoso!": etnografia dos cuidados a pacientes com hipertensão e/ou diabetes na atenção básica, em Manaus, Amazonas, Brasil. Cad Saúde Pública. 2008; 24(suppl.1): s91-s99. PMid:18660917. http://dx.doi.org/10.1590/S0102-311X2008001300014

5. Lima MT, Bucher JSNF, Lima JWO. A hipertensão arterial sob o olhar de uma população carente: estudo exploratório a partir dos conhecimentos, atitudes e práticas. Cad Saúde Pública. 2004; 20(4): 1079-1087. PMid:15300301. http://dx.doi.org/10.1590/S0102-311X2004000400023

6. Nissinen A, Berrios X, Puska P. Community-based noncommunicable disease interventions: lessons from developed countries for developing ones. Bull World Health Organ. 2001; 79(10): 963-970. PMid:11693979 PMCid:PMC2566669. 
7. Kulcar Z. Self-help, mutual AID and chronic patients' clubs in Croatia, Yugoslavia: discussion paper. J R Soc Méd. 1991; 84(5): 288-291. PMid:2041007 PMCid:PMC1293228.

8. Casanova F, Osorio LC, Dias LC. Abordagem comunitária: grupos na atenção primária à saúde. In: Gusso G, Lopes JMC, organizadores. Tratado de medicina de família e comunidade: princípios, formação e prática. Porto alegre: Artmed; 2012. v. 1, p. 265-273.

9. Diao D, Wright JM, Cundiff DK, Gueyffier F. Pharmacotherapy for mild hypertension. Cochrane Database Syst Rev. 2012; (8): CD006742. http:// dx.doi.org/10.1002/14651858.CD006742.pub8

10. Fontanella BJB, Ricas J, Turato ER. Amostragem por saturação em pesquisas qualitativas em saúde: contribuições teóricas. Cad Saúde Pública. 2008; 24(1): 17-27. PMid:18209831. http://dx.doi.org/10.1590/S0102-311X2008000100003

11. Bardin L. Análise de conteúdo. 3. ed. Lisboa: Edições 70; 2004.

12. Cotta RMM et al. Reflexões sobre o conhecimento dos usuários no contexto do Programa de Saúde da Família: a lacuna entre o saber técnico e o popular. Physis. 2008; 18(4): 745-766. http://dx.doi.org/10.1590/S0103-73312008000400008

13. Alves VS. Um modelo de educação em saúde para o Programa Saúde da Família: pela integralidade da atenção e reorientação do modelo assistencial. Interface: Comunic Saúde Educ. 2005; 9(16): 39-52. http://dx.doi.org/10.1590/S1414-32832005000100004

14. Cyrino AP, Schraiber LB. Promoção da saúde e prevenção de doenças: o papel da educação e da comunicação. In: Martins MA, Carrilho FJ, Alves VAF, Castilho EA, Cerri GG, Wen CL, organizadores. Clínica Médica, Barueri: Manole; 2009. v. 1, p. 470-477.

15. Rose G. Estratégias da Medicina Preventiva. Porto Alegre: Artmed; 2010.

16. Ferreira Neto JL, Kind L. Práticas grupais como dispositivo na promoção da saúde. Physis. 2010;20(4):1119-1142.

17. Freire P. Pedagogia do Oprimido. 17. ed. Rio de Janeiro: Paz e Terra; 1987.

18. Gomes LB, Merhy EE. Compreendendo a educação popular em saúde: um estudo na literatura brasileira. Cad Saúde Pública. 2011; 27(1): 7-18. http://dx.doi.org/10.1590/S0102-311X2011000100002

19. Vasconcelos EM. Educação popular nos serviços de saúde. 3. ed. São Paulo: Hucitec; 1997.

20. Ribeiro AG et al. Representações sociais de mulheres portadoras de hipertensão arterial sobre sua enfermidade: desatando os nós da lacuna da adesão ao tratamento na agenda da saúde da família. Physis. 2011; 21(1): 87-112. http://dx.doi.org/10.1590/S0103-73312011000100006

21. Tesser CD. Três considerações sobre a "má medicina". Interface: Comunic Saúde Educ. 2009; 13(31): 273-86.

22. Cyrino AP. Entre a ciência e a experiência: uma cartografia do autocuidado no diabetes. São Paulo: Ed. UNESP; 2009.

23. Haynes RB, Ackloo E, Sahota N, McDonald HP, Yao X. Interventions for enhancing medication adherence. Cochrane Database Syst Rev. 2008 Apr 16; (2): CD000011. http://dx.doi.org/10.1002/14651858.CD000011.pub3

24. Valla W. Educação popular, saúde comunitária e apoio social numa conjuntura de globalização. Cad Saúde Pública. 1999; 15(supl.2): 7-14.

25. Czeresnia D, Freitas CM. Promoção da saúde: conceitos, reflexões, tendências. Rio de Janeiro: Editora Fiocruz; 2003.

26. Carvalho SR. Os múltiplos sentidos da categoria "empowerment" no projeto de Promoção à Saúde. Cad Saúde Pública. 2004; 20(4): 1088-1095. PMid:15300302. http://dx.doi.org/10.1590/S0102-311X2004000400024

27. Santos LM, Da Ros MA, Crepaldi MA, Ramos LR. Grupos de promoção à saúde no desenvolvimento da autonomia, condições de vida e saúde. Rev Saúde Pública. 2006; 40(2): 346-352. http://dx.doi.org/10.1590/S0034-89102006000200024

28. Fernandes, MTO, Silva LB, Soares SM. Utilização de tecnologias no trabalho com grupos de diabéticos e hipertensos na Saúde da Família. Ciênc Saúde Coletiva. 2011; 16(suppl.1): 1331-1340. PMid:21503483. http://dx.doi.org/10.1590/S1413-81232011000700067 